\title{
Recovering Uniform Coverage in a 3D Survey: Case Study from Onshore Southern India
}

\author{
K. Shukla, ${ }^{1}$ P. Jaiswal, ${ }^{1}$ and C. S. Singh ${ }^{2}$ \\ ${ }^{1}$ Boone Pickens School of Geology, Oklahoma State University, Stillwater, OK 74078, USA \\ ${ }^{2}$ GAIL India Limited, New Delhi 110066, India \\ Correspondence should be addressed to K. Shukla; khemraj@okstate.edu
}

Received 28 January 2014; Revised 16 April 2014; Accepted 24 April 2014; Published 20 May 2014

Academic Editor: Alexey Stovas

Copyright (c) 2014 K. Shukla et al. This is an open access article distributed under the Creative Commons Attribution License, which permits unrestricted use, distribution, and reproduction in any medium, provided the original work is properly cited.

\begin{abstract}
We present a case study on 3D seismic acquisition under inaccessible ground conditions and heavy head-loads. The novelty in this case study is designing novel shot recovery strategies. The study area is in a densely populated region in Southern India, where a 3D survey was conducted over an area of $700 \mathrm{~km}^{2}$ to illuminate a target formation at $3000 \mathrm{~m}$ depth. The survey was designed in a brick pattern with six shots in a round, a line repeat interval of 2 , and offset and skid grid of $300 \mathrm{~m} \times 300 \mathrm{~m}$. Only $40 \%$ of full fold could be obtained with conventional shot recovery methods. To increase the survey fold, two new shot recovery strategies were attempted. First, the original offset and skid grid was increased to $1100 \mathrm{~m} \times 1200 \mathrm{~m}$. Second, if the recovery shot did not fall in this grid, it was relocated along the swath from its original location to a distance equal to half the inline offset. The two strategies, employed together, increased the fold to $90 \%$ of full fold at the target area maintaining its uniformity. Although the illumination of the target zone was adequate for interpretation in this case, we see a need for adaptation if these strategies are to be applied in other surveys. In general, issues related to offset-limit, shot-density, and grid-spacing are a matter of continual optimization in 3D surveys.
\end{abstract}

\section{Introduction}

Since its advent, 3D seismic acquisition has had a substantial impact on hydrocarbon exploration [1]. 3D surveying provides many benefits over $2 \mathrm{D}$ but it presents challenges that are unique and often require solutions based on local geology and resource availability. 3D acquisition cannot be treated as a mere extension of 2D; it is distinguished by simultaneous data recording along multiple, closely-spaced broadside profile which enables reconstruction of the true nature of the seismic wavefield. As opposed to 2D, 3D acquisition creates a data volume from which lines, planes, or probes can be extracted in any orientation [2]. While it is possible to acquire a dense, high-resolution grid of $2 \mathrm{D}$ lines or create a mesh of $2 \mathrm{D}$ coverage by assembling spatially coincident $2 \mathrm{D}$ surveys of different vintages [3], unless the data are sampled in 3D, spatial aliasing is difficult to circumvent [4].

The basic assumption of designing a 3D survey is single plane layer over a half space [5]. The most critical parameter in a $3 \mathrm{D}$ survey design is the bin size, which determines the maximum wavenumber sampled in the field which then sets the threshold for spatial aliasing $[6,7]$. It is computed as

$$
\Delta x \leq \frac{v}{4 f \sin \theta},
$$

where $v$ is average velocity up to the target depth, $f$ is the dominant frequency at the target depth, and $\theta$ is the dip of the target formation [8]. In essence, (1) implies that the bin size should be less than or equal to one-fourth of the surface component of the dominant wavenumber. Although in theory only higher-than-dominant frequencies are at risk of spatial aliasing, in practice setting bin size according to the highest frequency invokes data redundancy. Liner et al. [9] proposed an alternate form of (1) for stratigraphy with rapidly changing velocity gradients:

$$
\Delta x \leq \frac{1}{4 f p} .
$$

In (2), $p$ is the ray parameter (horizontal slowness) which stays constant along the ray path. 
The bin size is an outcome of shot and receiver spacing. In $3 \mathrm{D}$, the receiver can be laid out in grids. The spacing of the receivers along one direction is usually less than the other; this direction is the "Inline" directions and the perpendicular direction is the "Crossline" directions. Traditionally both shots and receiver are laid out in linear arrays; the intervals between respective arrays are referred to as shot line interval (SLI) and receiver line interval (RLI). Other important design parameters are minimum and maximum source-receiver offset, $X_{\min }$ and $X_{\max }$, respectively, which is set to provide adequate offsets for move-out analysis on shallowest and deepest targets. As a rule of thumb, $X_{\text {min }}$ should be small enough to allow adequate ray coverage of the shallowest reflector of interest that might be used for datuming. As opposed to $2 \mathrm{D}$, the $X_{\min }$ does not stay constant throughout the survey; it changes with the azimuth. The bin at the center of the box, formed by two adjacent receiver lines and two source adjacent lines, has the largest $X_{\text {min }}$ of any bin within the box. In a conventional orthogonal design (receiver lines and shot lines are perpendicular to each other) a typical $X_{\min }$ value is determined as [10]

$$
X_{\min }=\left[\mathrm{RLI}^{2}+\mathrm{SLI}^{2}\right]^{1 / 2}
$$

Besides parameterization complexity, 3D design also needs to address ground realities-costs versus survey size versus structural dip at the depth-of-interest, need of more complex static solutions, and higher susceptibility to culture to name a few $[11,12]$. The latter in particular, which is also the focus of this paper, becomes more pronounced in urban areas. Terrain inaccessibility due to cultural establishments in urban areas can create survey gaps. As a result, shots have to be skipped which makes the survey fold nonuniform and unequally distributed (both in offset and azimuth) creating suboptimal datasets for processing and imaging [13]. Fomel [14] demonstrates how irregular trace spacing and survey gaps lead to migration artifacts.

To some extent, data gaps in 3D can be compensated through interpolation, for example, sparseness constrained least square inversion [15], minimum weighted norm interpolation [16], and simultaneous interpolation of four spatial dimensions [17]. The interpolation is strictly meant to prepare data for imaging, which, under ideal conditions, require all offset ranges to be present within all CMPs. Interpolation cannot recover data that was not sampled in the field. Thus, it is more desirable to design compensation strategies in the field by rearranging the shots and receivers such that the adequate ray coverage at the target depth is achieved. Cordsen et al. [18] summarize three ways in which skipped shots are generally compensated for based on the survey priority. These include the following: (a) if maintaining fold in each bin is the priority, move the source by less than half the group interval along the inline direction; (b) if maintaining offset distribution in each bin is a priority, move the source up to half the SLI; and (c) if populating a bin is the priority, then skid (moving shots in the crossline direction) [19]. On a related note, skidding unbalances the symmetry in a spilt spread and therefore is adopted only as a last resort when data in skipped shot positions cannot be compensated in any other manner.

The survey discussed in this paper is conducted in a densely populated region of Southern India (Figure 1). Due to the size and orientation of the cultural establishments, none of the rule of thumb shot-receiver rearrangements described above could achieve more than $40 \%$ of the full fold (fold obtained if no surface obstacles were present). Two new recovery strategies were conceived which increase the fold to $90 \%$ of the full fold; this paper discusses their field implementation. This paper has the following structure. First, we introduce the study area. Next, we show survey parameterization and introduce the new recovery strategies. Following this, we compare and contrast the survey fold obtained from our recovery strategies with survey fold obtained from the strategy above with maintaining fold in each bin as the priority. Finally, we discuss the current limitations and potential improvements in the offset-recovery method.

\section{Study Area and Survey Design}

The study area is in the onshore part of the Cauvery Basin located between the latitudes $8^{\circ} 30^{\prime}$ and $12^{\circ} 30^{\prime} \mathrm{N}$ and longitudes $78^{\circ} 30^{\prime} \mathrm{E}$ and $80^{\circ} 20^{\prime} \mathrm{E}$ in the Indian peninsula (Figure 1). The survey area is densely populated and heavily cultivated and covered by a closed grid of road networks (Figure 1(b)). Previously, exploration targets have been identified using 2D profiles (Figure 2). The main objective of the $3 \mathrm{D}$ survey was to illuminate (obtain a full fold) targets at the depth range of 2500-300 $\mathrm{m}$. The survey was designed in a brick pattern [20], which is a type of orthogonal layout where either the source or receiver lines or both are staggered. The brick layout is advantageous because (a) it gives adequate space for skidding and (b) the largest $X_{\text {min }}$ is minimum for given SLI and RLI [18]. Using a nominal velocity profile developed using $2 \mathrm{D}$ data $(500 \mathrm{~m} / \mathrm{s}$ at the surface increasing to $3500 \mathrm{~m} / \mathrm{s}$ at the target depth), a $25 \mathrm{~m} \times 25 \mathrm{~m}$ bin size was estimated [21].

The $X_{\max }$ in the survey depends on the depth of interest in imaging but there is an inherent tradeoff between $X_{\text {min }}$ and $X_{\max }$. For velocity analysis, a survey aperture of at least $30^{\circ}$ is recommended at the target zone [22]. However, large offsets intensify NMO stretching. To avoid the effect of NMO stretching on stack, the shallow data are generally muted which degrades the quality of the stack towards shallower time/depth. Thus, in the end, there is a trade-off between the deepest horizon which can be conventionally stacked and the offset available for stacking the shallow horizons. In survey designs, it is common to assume that at least four traces contain reflections from the shallowest horizon of interest for stacking after muting the NMO stretch. Thus, $X_{\max }$ needs to be chosen such that the mute cone with a maximum sourcereceiver offset denoted as $X_{\text {mute }}$ allows for this. Further, increasing $X_{\max }$ may increase the survey cost and if the same receiver group is spread out more generously, it will decrease the fold at the zone of interest [23]. Vermeer [24] 


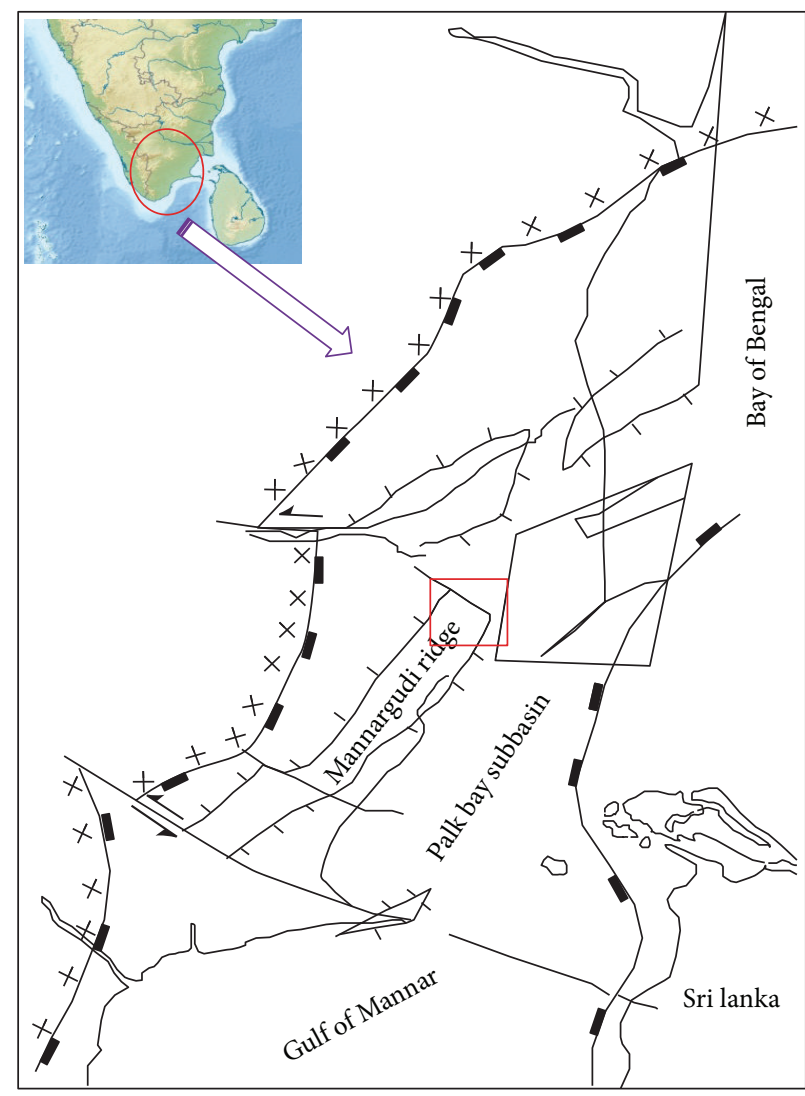

(a)

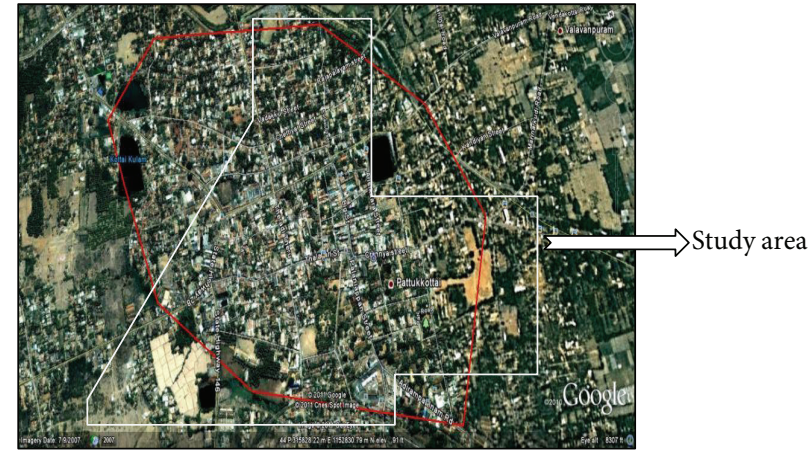

(b)

FIGURE 1: Location map. (a) Study area in blue polygon plotted on backdrop of tectonic map. (b) Study area in backdrop of demographic distribution.

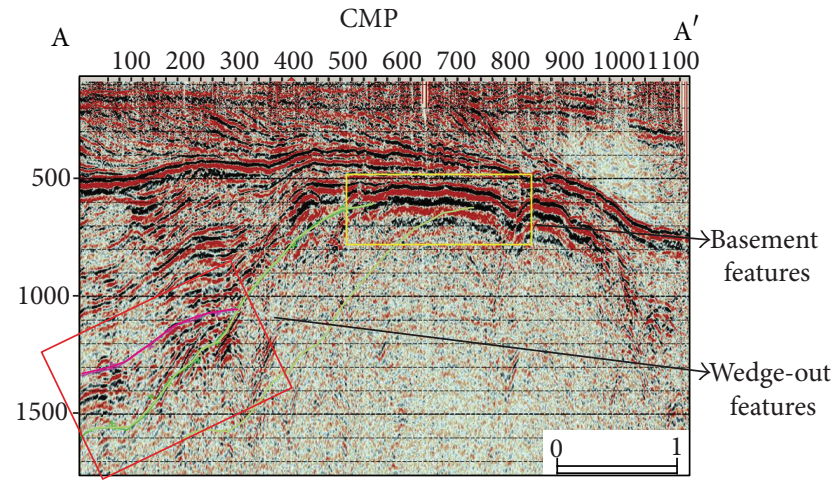

FIGURE 2: Target formations to be illuminated in 3D seismic survey.

provided an empirical relation between $X_{\min }$, SLI, and RLI for symmetric sampling in a brick geometry as

$$
X_{\min }=\mathrm{RLI} * \sqrt{1.25} .
$$

In our case study, the datum is $\sim 50 \mathrm{~m}$ below the topography. To fulfill the criteria mentioned in (3), SLI and RLI were chosen (Table 1) such that $X_{\min }$ is $500 \mathrm{~m}$, which satisfies the criteria of the fourfold sampling at shallowest target.

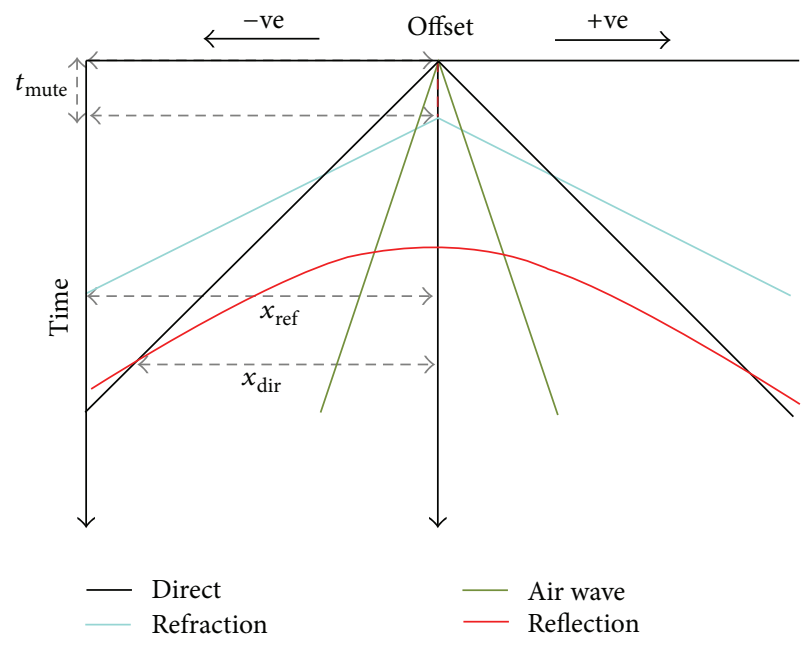

FIgURE 3: Seismic preprocessing gates.

The $X_{\max }$ was kept equal to the target depth (such that the aperture is $\sim 30^{\circ}$ ). Interference from direct wave (Figure 3) was also considered in estimating $X_{\max }$ in the following manner [25]. Assuming that the direct wave starts interfering 


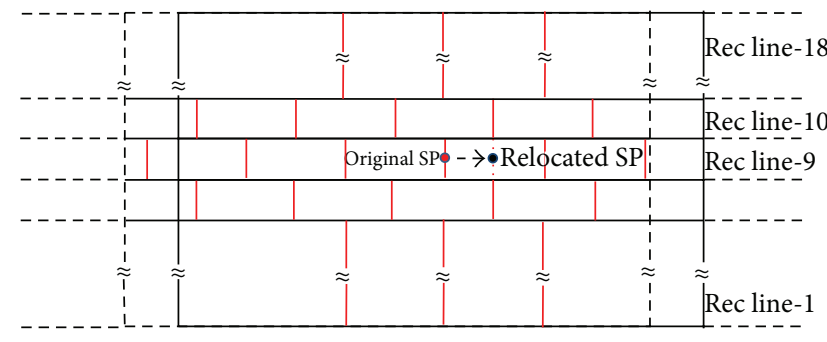

$\square$ Template for original shot point

ㄷ. Template for relocated shot point

(a)

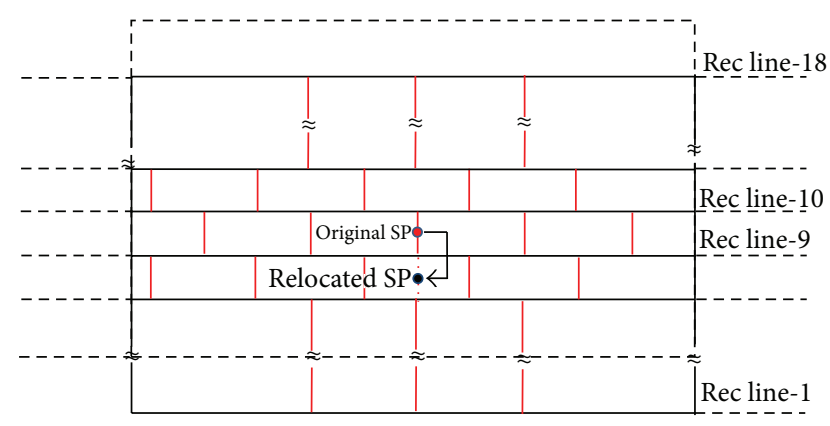

Template for original shot point ¿ $\sqsupset$ Template for relocated shot point

(b)

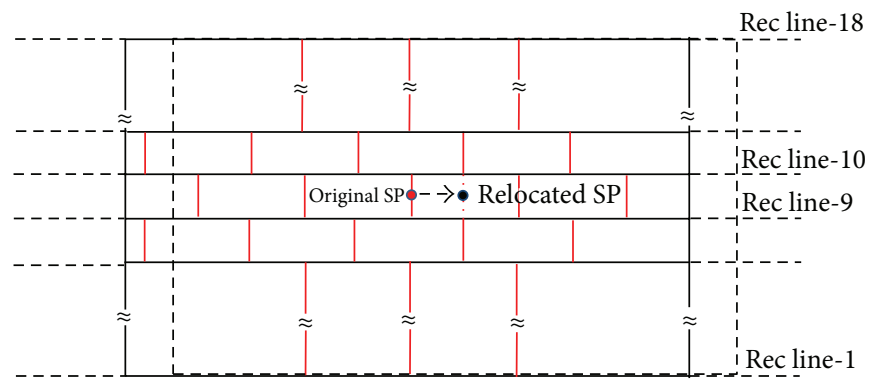

Template for original shot point

ここ Template for relocated shot point

(c)

FIGURE 4: Offset-recovery methods. (a) Shot recovered at offset perpendicular to the shot-line with template moving in opposite direction. (b) Shot recovered at offset along the shot-line with template moving in opposite direction. (c) Relocation of the shots in the same swath with new template.

with the primary reflection at an offset $X_{\text {dir }}$ and move out time $t_{\mathrm{NMO}}, X_{\mathrm{dir}}$ and $t_{\mathrm{NMO}}$ are expressed as

$$
\begin{gathered}
X_{\mathrm{dir}}=V_{\mathrm{LVL}} *\left(t_{\mathrm{NMO}}+t_{\mathrm{mute}}\right), \\
t_{\mathrm{NMO}}=\left(t^{2}+\frac{X_{\mathrm{dir}}^{2}}{V^{2}}\right) .
\end{gathered}
$$

In (5), $V_{\mathrm{LVL}}$ is the datuming velocity, $V$ is the stacking velocity at the target depth, and $t_{\text {mute }}$ is the intercept of the first reflection, above which all data are muted. In our case, $X_{\max }$ is considered to be smaller than $X_{\mathrm{dir}}, V_{\mathrm{LVL}}$ is computed using up-hole survey as $1850 \mathrm{~m} / \mathrm{s}, V$ is computed using vintage $2 \mathrm{D}$ seismic as $2500 \mathrm{~m} / \mathrm{s}$, and $t_{\text {mute }}$ is $180-220 \mathrm{~ms}$ based on visual inspection of the shot.

\section{Recovery Strategy and Results}

Acquisition parameters for the 3D survey are given in Table 1. The following nontraditional recovery strategies were used:

(1) extending the maximum offset and recovery arms by one SLI and two RLI, respectively, and subsequently moving the original designed template (zone of active receivers) in opposite direction of movement of shots (Figures 4(a) and 4(b));
TABLE 1: Acquisition parameters of the survey.

\begin{tabular}{lc}
\hline \multicolumn{2}{c}{ Acquisition parameters } \\
Basic parameters & Parameters value \\
\hline Template & $120 \mathrm{Ch} \times 18$ Receiver lines \\
Receiver interval & $50 \mathrm{~m}$ \\
Receiver line interval & $300 \mathrm{~m}$ \\
Source point interval & $50 \mathrm{~m}$ \\
Source line interval & $600 \mathrm{~m}$ \\
Bin size & $25 \mathrm{~m} \times 25 \mathrm{~m}$ \\
Nominal fold & 45 \\
\hline
\end{tabular}

(2) if shots cannot be accommodated in the extended offset-recovery grid, then they were placed along the same swath with a maximum distance equal to half of the maximum inline RLI offset; templates for these shots were redesigned considering them as new shots (Figure 4(c)).

We compare our strategies with the conventional strategies through simulations from MESA $\odot$, a popular field design software. We simulate ray coverage at the surface (Figure 5), ray coverage at the target level (Figure 6), fold versus offset distribution (Figure 7), and total fold of the survey (Figure 8) 


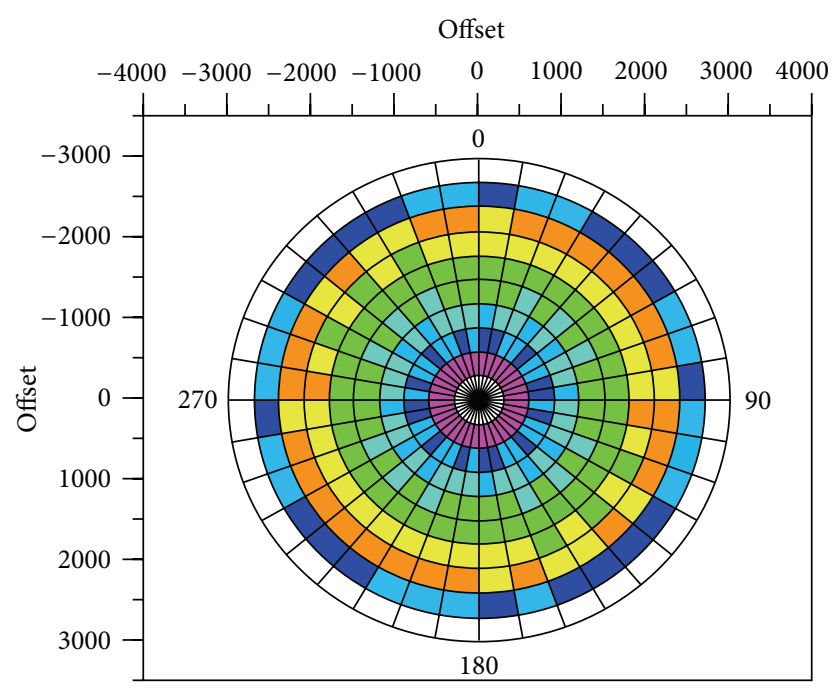

(a)

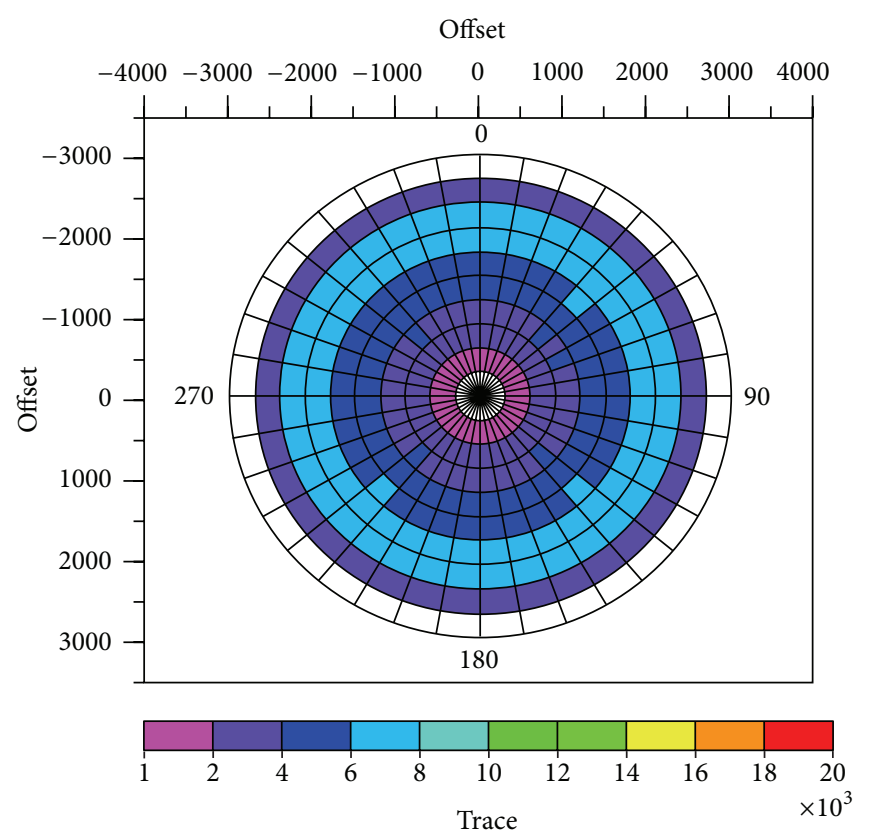

(c)

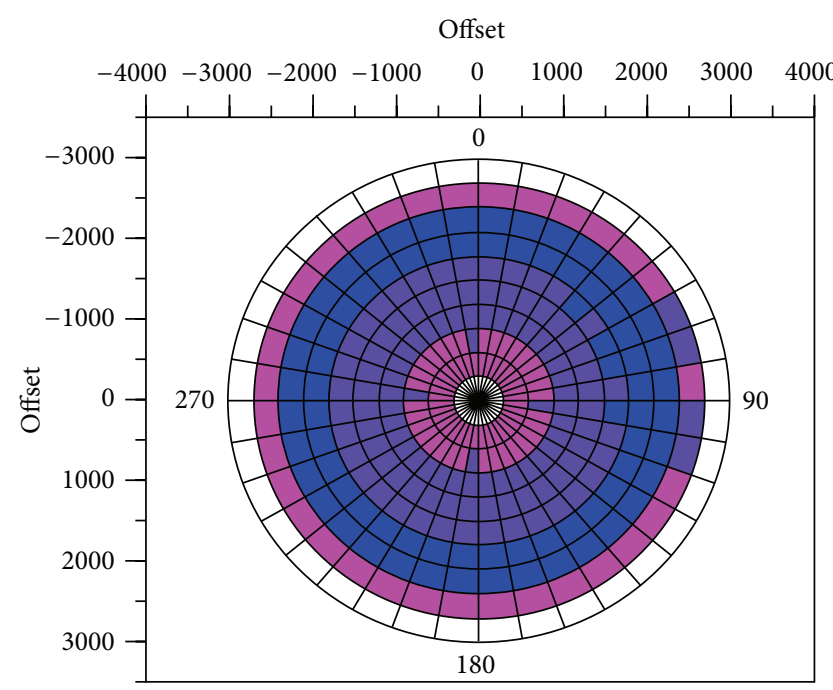

(b)
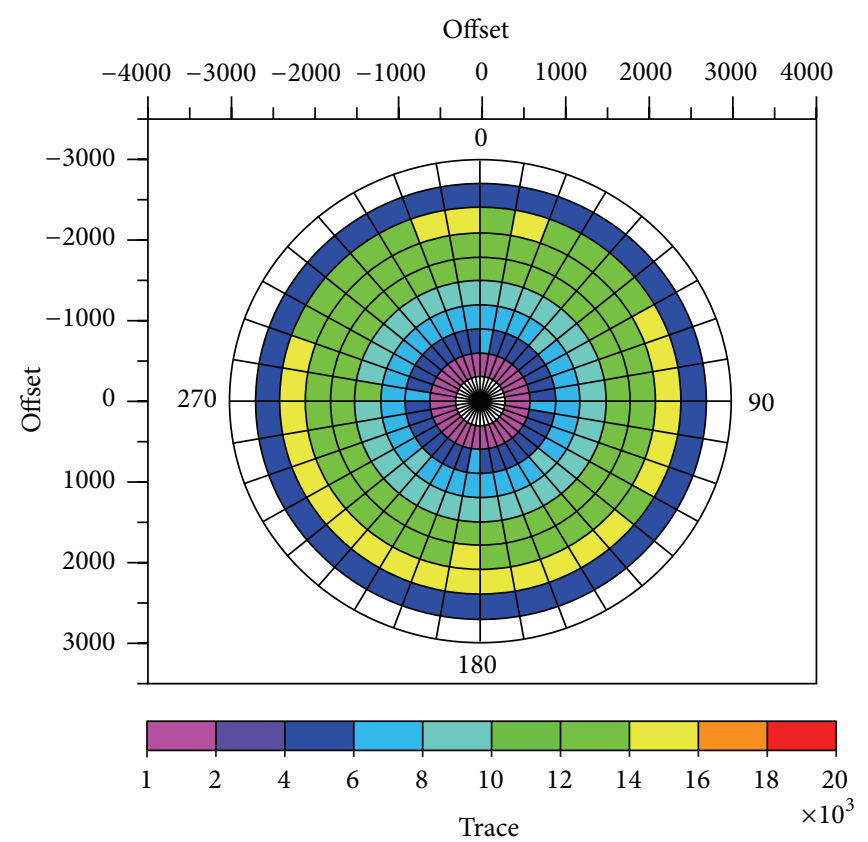

(d)

FIGURE 5: Total ray coverage in (a) acquisition with no cultural noise, (b) acquisition with real cultural noise, (c) traditional recovery method applied on (b), and (d) new recovery strategies applied on (c).

for four cases: (i) full fold, (ii) with field-surface obstacles and no recovery, (iii) using conventional recovery with a priority of maintaining regular fold in every bin, and (iv) using the two novel strategies described in the section above in addition to (iii). Figures 5 and 6 clearly show that the surface ray coverage and the ray coverage at target depth are close to the ray coverage in the full fold case when the new recovery strategies are employed. Figures 5(d) and 6(d) show that there is an increase in $X_{\max }$ at a few azimuths; the fold corresponding to these bins is low enough that the bins can be ignored for velocity analysis. Figure 6 also shows that the fold at $X_{\min }$ is 7 , which is greater than the minimum number of traces needed for velocity analysis.

Figure 7 shows that the fold distribution after the application of new strategies is close to full fold, which is the biggest achievement. Due to the increase in azimuthal coverage from rearrangement of shots, an increase of fold at offsets equal to the target depth as well as at the near offsets $(0-200 \mathrm{~m})$ was observed. However these small increments in fold are not significant to help in constraining velocity any better. After the application of new strategies, the fold of the survey remains fairly uniform (Figure 8) except at locations where 


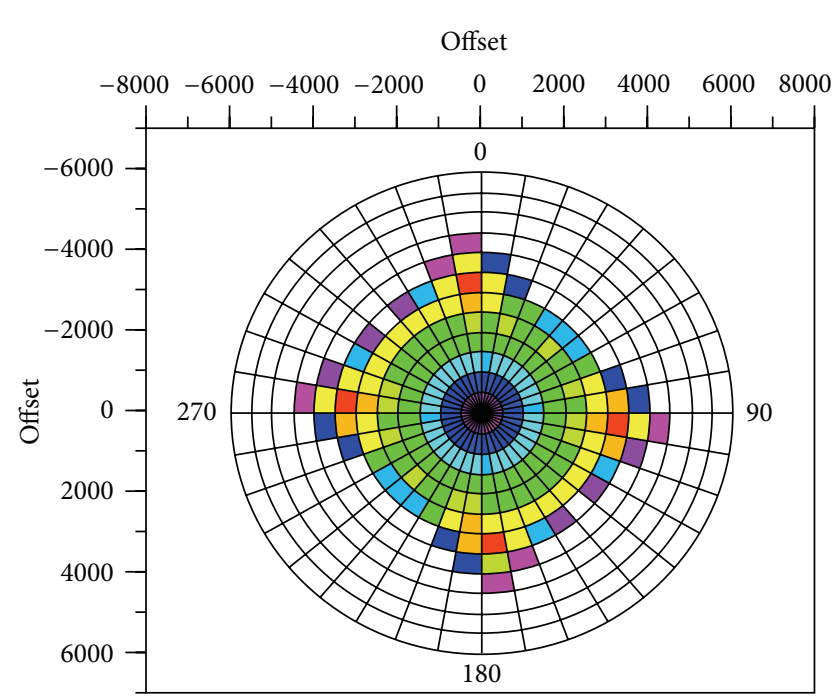

(a)
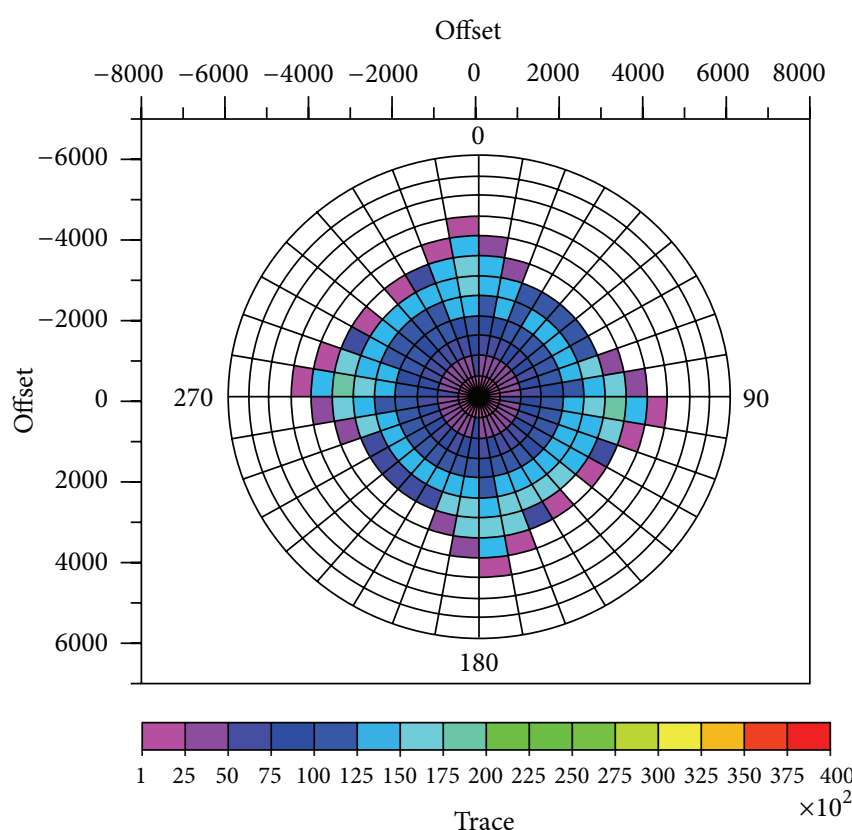

(c)

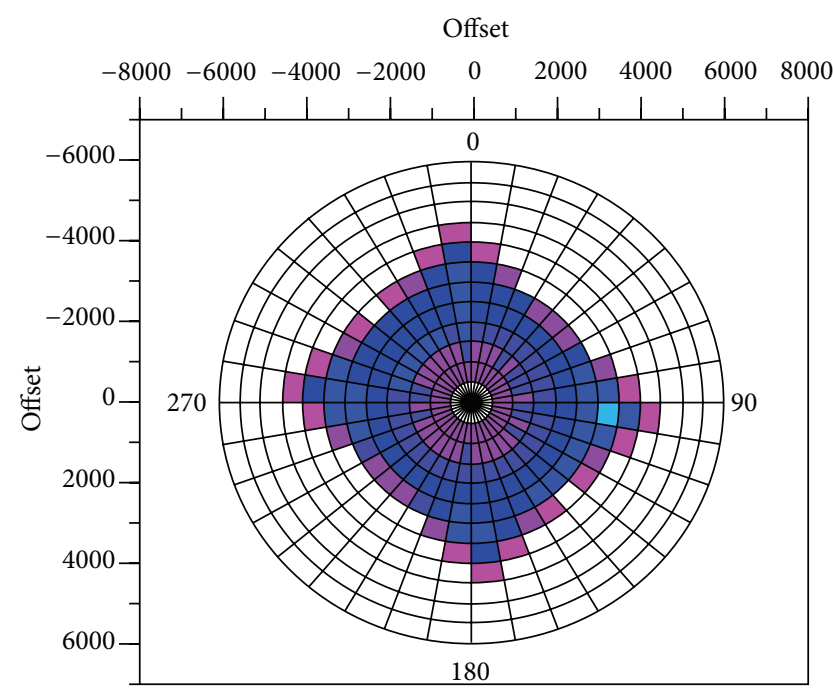

(b)
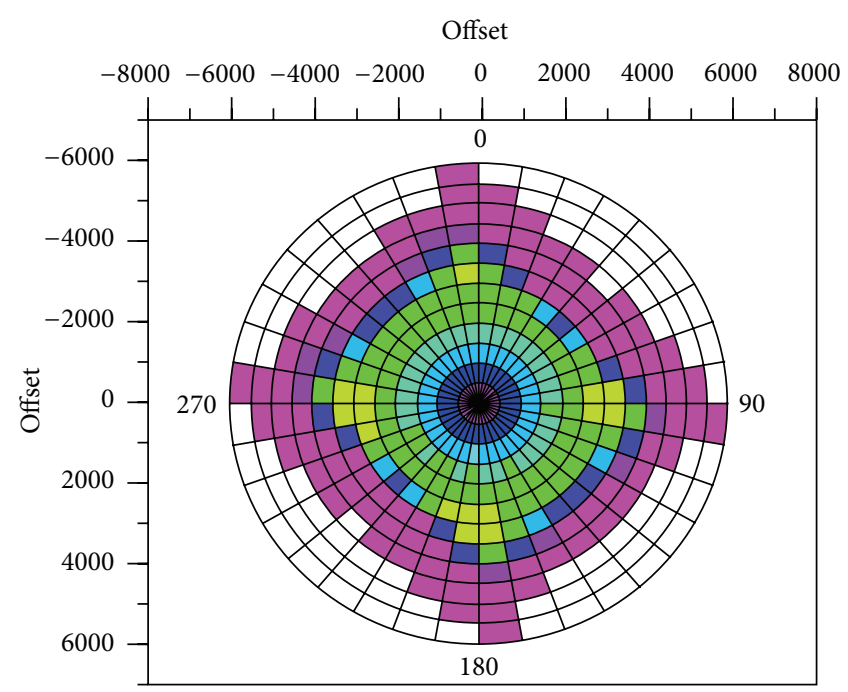

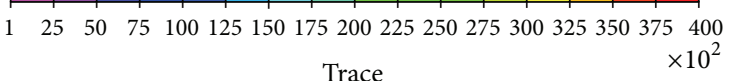

(d)

FIGURE 6: Total ray coverage at target levels in (a) acquisition with no cultural noise, (b) acquisition with real cultural noise, (c) traditional recovery method applied on (b), and (d) new recovery strategies applied on (c).

the shots are relocated; shot relocation causes a fold increase on one side of the template while reducing it on the other side. Most notable is the achievement of fold of 36 at the current level below the inaccessible ground location, which in the absence of the new strategies was zero.

\section{Discussions}

The new strategies proposed in this paper have many dependencies and are conditional. First, the shooting pattern should be single swath for more regular offset distribution as opposed to multiple-swath shooting [18]. In 3D surveys, binning creates data discontinuities as it breaks a continuous wavefield into locally concentrated reflection points that eventually manifests as acquisition footprints. In the brick layout, the largest $X_{\min }$ is the least in any possible orthogonal design, which improves offset and azimuth distribution at shallow levels while minimizing the acquisition footprints at the same time. However, after application of our strategies, the recovered and skidded shots may reduce offset and azimuth distribution at shallow levels, which could intensify the footprint problem. We emphasize that in the brick pattern the computation of largest $X_{\min }$ is not directly due to staggering of the source lines. As a result for each recovered 


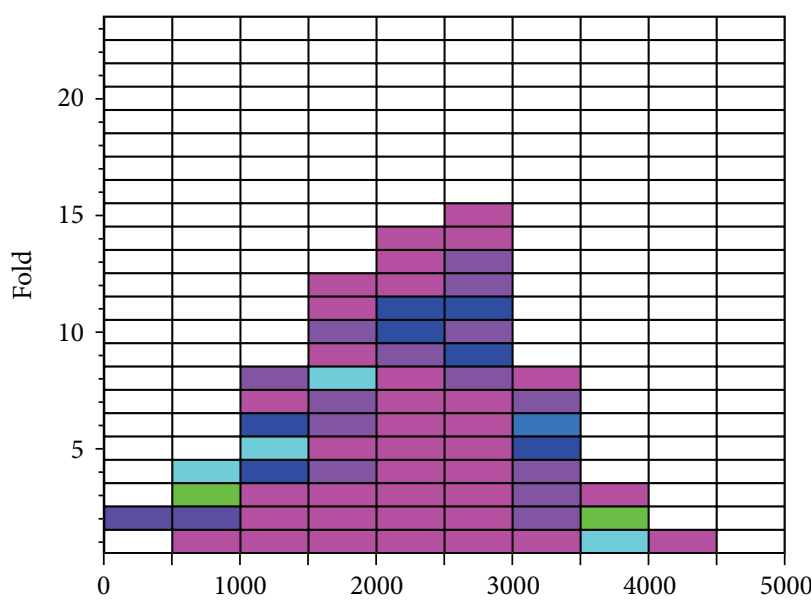

(a)

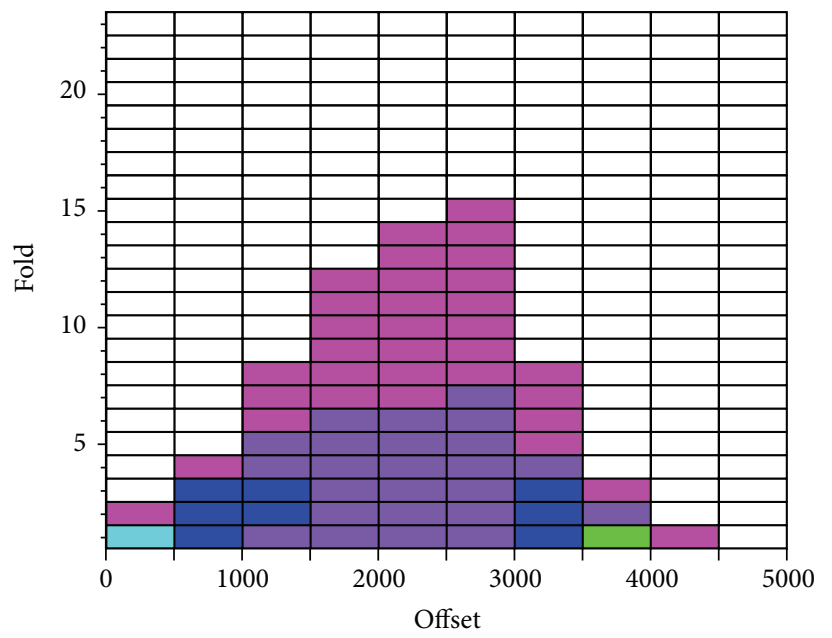

(Traces)
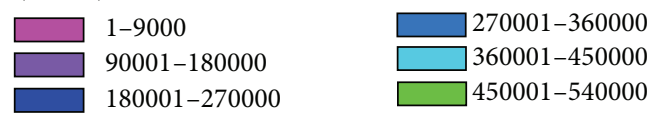

(c)

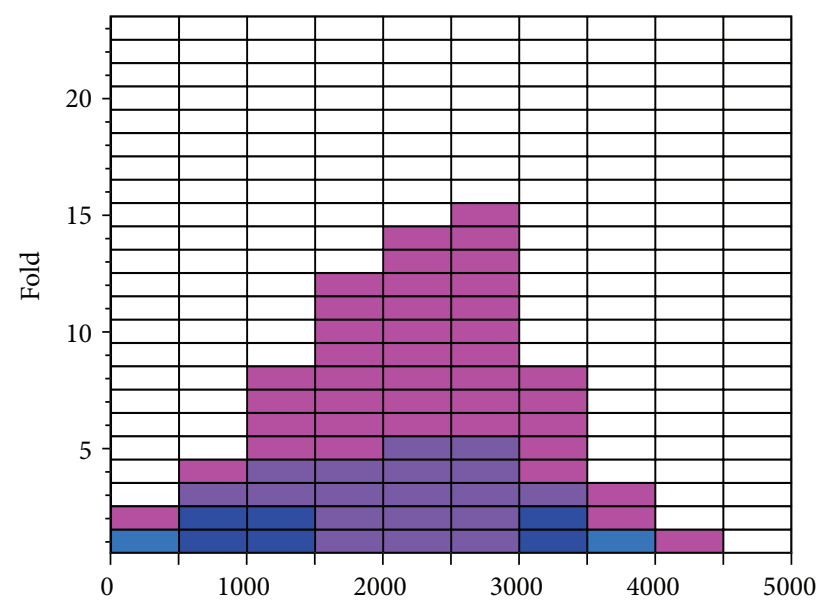

(b)

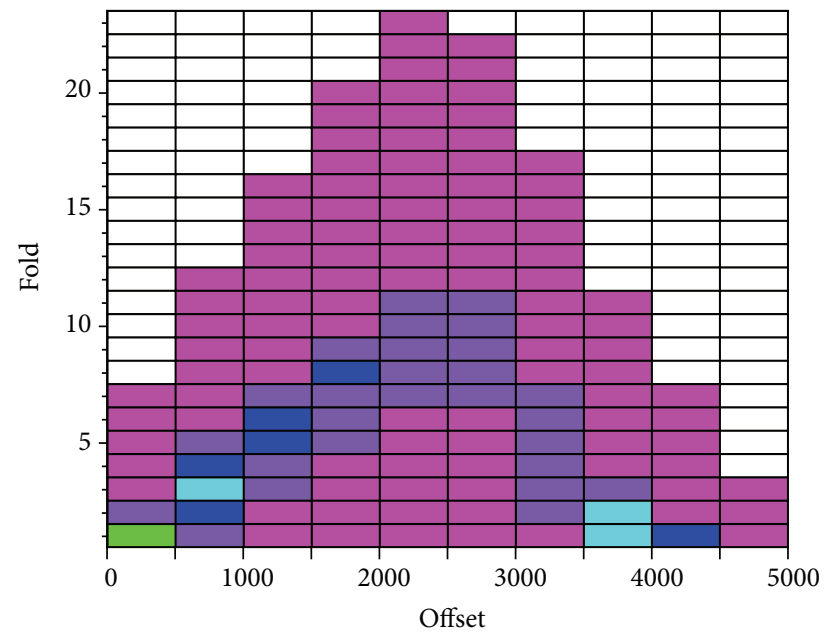

(Traces)

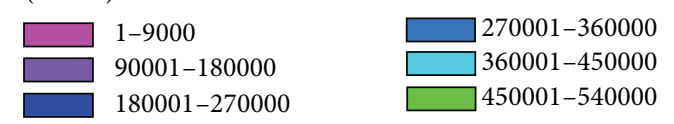

(d)

FiguRE 7: Fold versus offset distribution in (a) acquisition with no cultural noise, (b) acquisition with real cultural noise, (c) traditional recovery method applied on (b), and (d) new recovery strategies applied on (c).

and skidded shots, the offsets and azimuth distribution at target levels need to be confirmed by simulations.

Implementation of our strategies requires a detailed geodetic survey. Exercising these methods with proper planning, which includes preparation of a new geometry for rearranged shots and some additional surveys, will result in obtaining the maximum possible coverage of a planned survey in an area of difficult logistics. The proposed strategies require a significant book keeping. Not only more ground equipment and regrouping of geophones arrays are required on a regular basis, the source-receiver layout also has to be dynamically updated (using Digital Global Positioning System). Application of these methods may not be economical in high terrain area due to logistic issues, the most important being cable connections that have to be altered. However, if the proposed recovery strategies are implemented with cable-less technology [26], a substantial amount of time can be saved. Although advantageous, these strategies require meticulous planning and conservation by means of simulation. Otherwise, not only they may result in delay of project completion but also cost increase.

\section{Conclusions}

Two novel field strategies of shot recovery within a 3D survey grid with inaccessible locations are presented. These are (a) generating a new template by extending the offset and recovery grid up to one SLI and two RLI and (b) relocating the shot up to maximum inline offsets with the new templates. In our study area which is a densely populated zone in Southern India, the new shot recovery strategy resulted in achieving $90 \%$ of full fold as opposed to the conventional recovery 


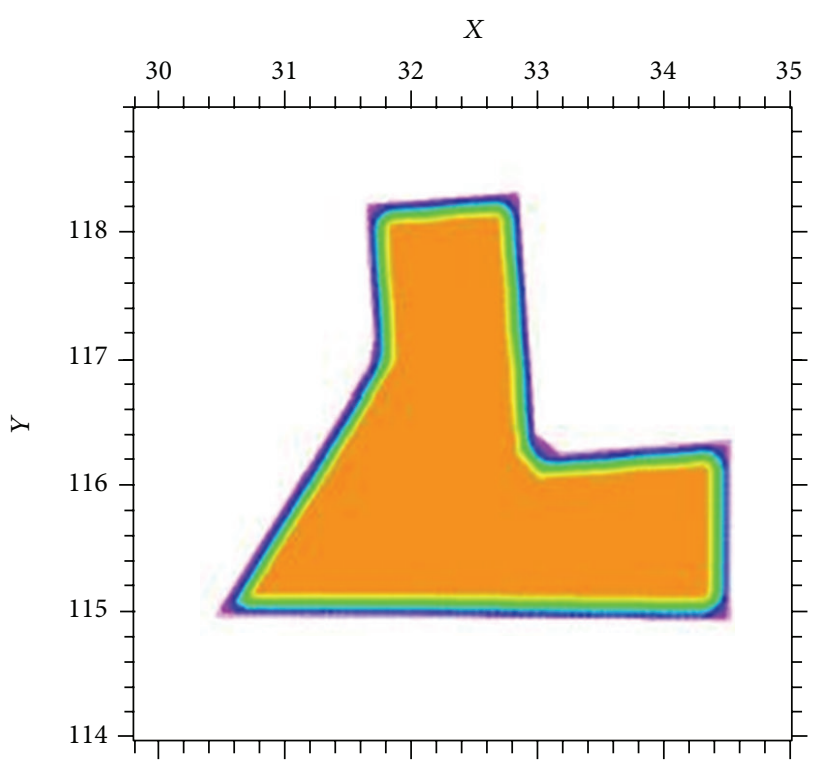

(a)

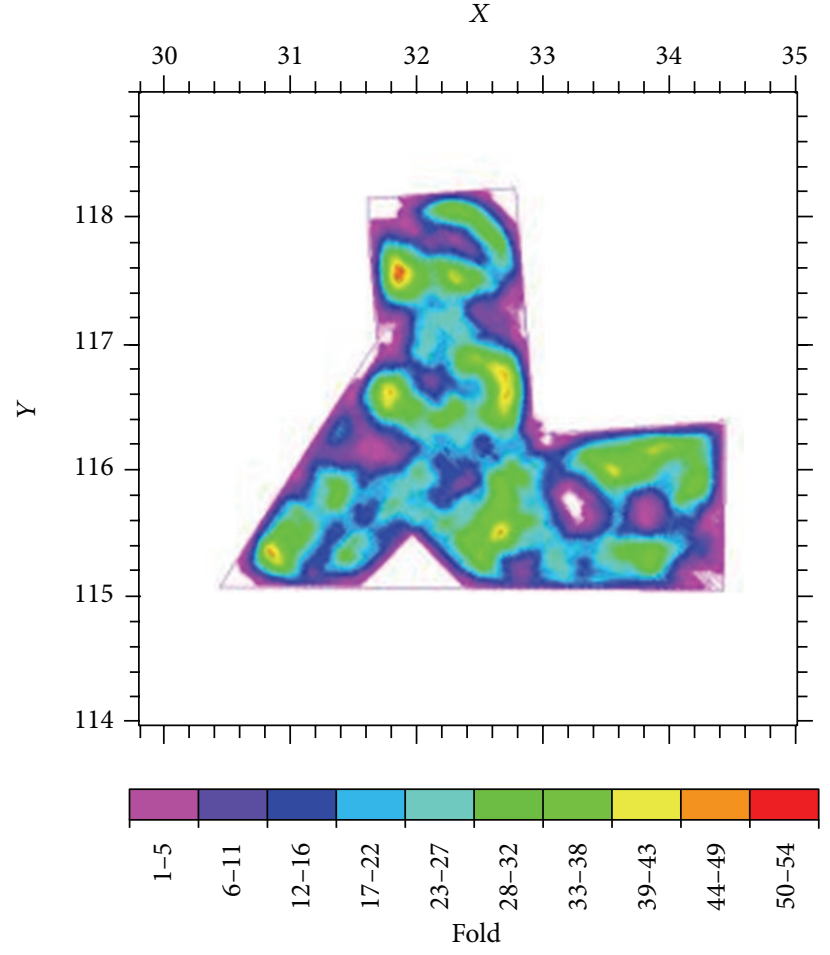

(c)

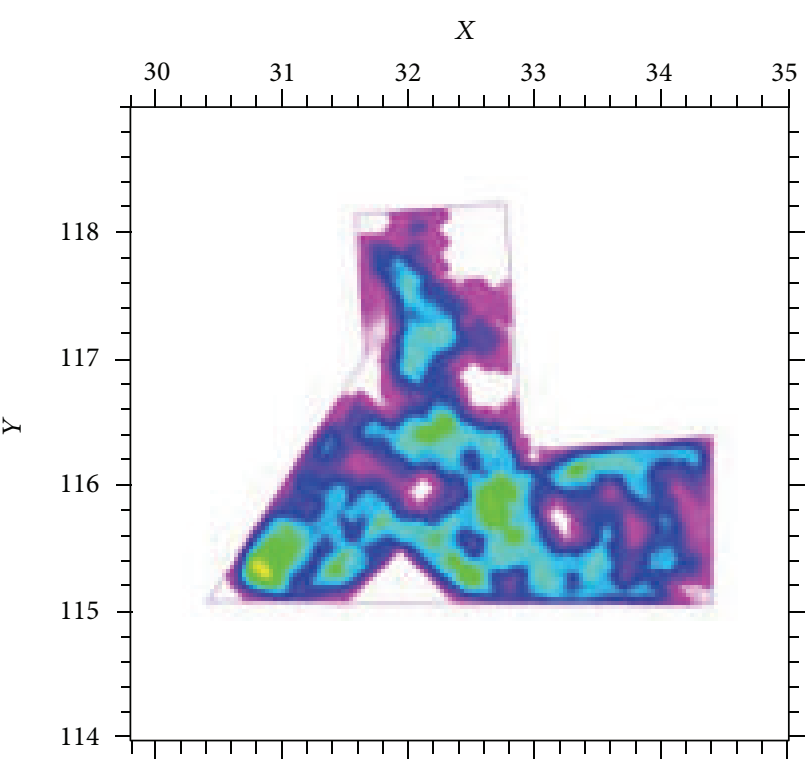

(b)

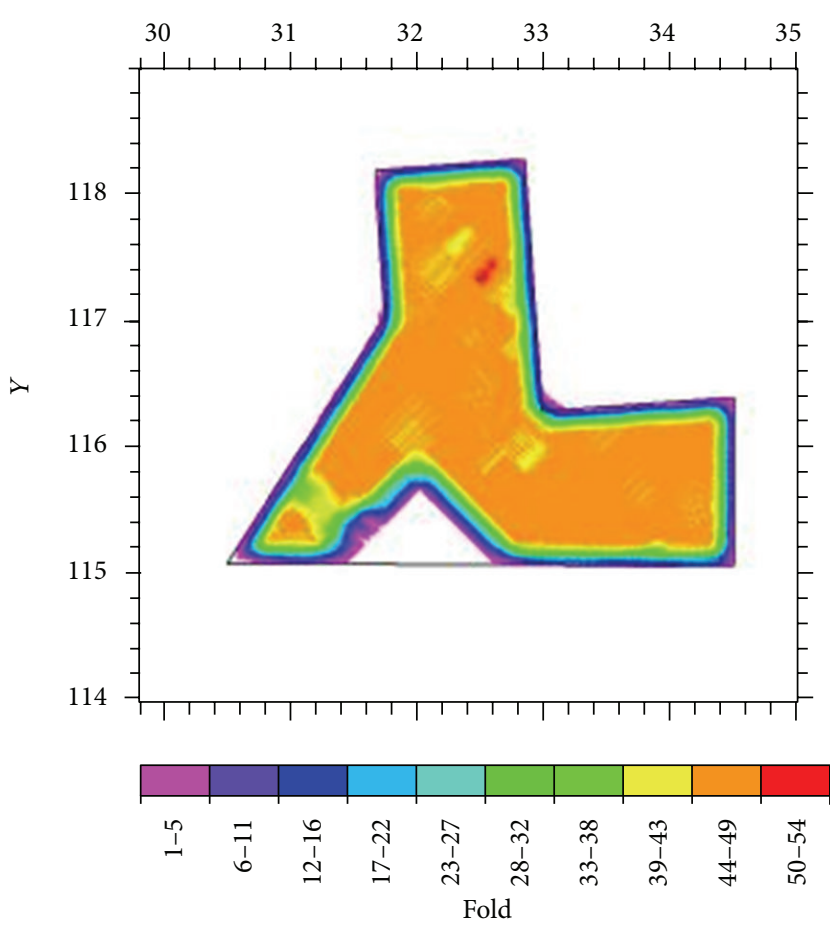

(d)

FIGURE 8: Total fold map in (a) acquisition with no cultural noise, (b) acquisition with real cultural noise, (c) traditional recovery method applied on (b), and (d) new recovery strategies applied on (c).

method which could only yield up to $30 \%$ of the full fold. A notable achievement was to obtain uniform fold coverage at the target level. On the downside, uneven fold distribution at the shallow subsurface which could potentially enhance the acquisition footprints could not be avoided. A combination of brick layout pattern along with our recovery methods could be very effective in populated urban areas; however they must be adapted to suit the needs of other 3D surveys.

\section{Conflict of Interests}

The authors declare that there is no conflict of interests regarding the publication of this paper.

\section{Acknowledgments}

The authors gratefully acknowledge the Boone Pickens School of Geology, Oklahoma State University, for 
providing the resources to conduct this study and the efforts of anonymous reviewer for bringing the paper at the publication level. The authors also acknowledged the editor and entire publication team of International Journal of Geophysics for their unparalleled effort to expedite the publication of this research. This is Boone Pickens School of Geology contribution number 2014-13.

\section{References}

[1] Y. Chingyong, "Full 3D seismic interpretation," Progress in Geophysics, vol. 3, article 014, 1999.

[2] R. J. Davies, S. A. Stewart, J. A. Cartwright et al., "3D seismic technology: are we realising its full potential?" Geological Society of London's Memoirs, vol. 29, no. 1, pp. 1-10, 2004.

[3] L. Lonergan and N. White, "Three-dimensional imaging of a dynamic Earth," in Visions of the Future: Astronomy and Earth Science, vol. 1, p. 157, Cambridge University Press, 2001.

[4] Ö. Yilmaz, Seismic Data Analysis: Processing, Inversion, and Interpretation of Seismic Data, SEG Books, 2001.

[5] C. P. Ashton, B. Bacon, C. Deplante, T. Sinclair, and G. Redekop, "3D seismic survey design," The Leading Edge, vol. 12, no. 11, pp. 1094-1117, 1993.

[6] J. F. Claerbout, Imaging the Earth's Interior, Blackwell Scientific Publications, 1985.

[7] Ö. Yilmaz and S. M. Doherty, Seismic Data Processing, Society of Exploration Geophysicists, 1987.

[8] M. T. Reblin, G. G. Chapel, S. L. Roche, and C. Keller, "Seismic interpretation, 28, A 3-D reflection seismic survey over the Dollarhide Field, Andrews County, Texas," The Leading Edge, vol. 10, no. 8, pp. 11-15, 1991.

[9] C. L. Liner, W. D. Underwood, and R. Gobeli, "3-D seismic survey design as an optimization problem," The Leading Edge, vol. 18, no. 9, pp. 1054-1060, 1999.

[10] D. G. Stone, Designing Seismic Surveys in Two and Three Dimensions, Volume 5, Society of Exploration Geophysicists, 1994.

[11] D. Trad, "Five-dimensional interpolation: recovering from acquisition constraints," Geophysics, vol. 74, no. 6, pp. V123V132, 2009

[12] M. J. G. Cox, E. F. Scherrer, and R. Chen, Static Corrections for Seismic Reflection Surveys, Volume 9, Society of Exploration Geophysicists, 1999.

[13] D. Trad, J. Deere, and S. Cheadle, "Understanding land data interpolation," in Proceedings of the 2005 SEG Annual Meeting, 2005.

[14] S. Fomel, Three-dimensional seismic data regularization [Ph.D. thesis], Stanford University, 2001.

[15] Y. Wang, "Sparseness-constrained least-squares inversions: application to seismic wave reconstruction," Geophysics, vol. 68, no. 5, pp. 1633-1638, 2003.

[16] B. Liu and M. D. Sacchi, "Minimum weighted norm interpolation of seismic records," Geophysics, vol. 69, no. 6, pp. 1560-1568, 2004.

[17] B. Liu, M. D. Sacchi, and D. Trad, "Simultaneous interpolation of 4 spatial dimensions," in Proceedings of the 2004 SEG Annual Meeting, 2004.

[18] A. Cordsen, M. Galbraith, and J. Peirce, Planning Land 3-D Seismic Surveys, Volume 9, Society of Exploration Geophysicists, 2000.
[19] T. W. Donze and J. Crews, "Moving shots on a 3-D seismic survey. The good, the bad, and the ugly (or how to shoot seismic without shooting yourself in the foot!)," The Leading Edge, vol. 19, no. 5, pp. 480-483, 2000.

[20] G. J. O. Vermeer and C. J. Beasley, 3-D Seismic Survey Design, Volume 12, Society of Exploration Geophysicists, 2002.

[21] C. L. Liner and W. D. Underwood, "3-D seismic survey design for linear v (z) media," Geophysics, vol. 64, no. 2, pp. 486-493, 1999.

[22] M. Galbraith, "A new methodology for 3D survey design," The Leading Edge, vol. 23, no. 10, pp. 1017-1023, 2004.

[23] A. Cordsen, "How to find the optimum 3D fold," in Proceedings of the 1995 Annual Meeting of the Canadian Society of Exploration Geophysicists, Expanded Abstract, pp. 96-97, 1995.

[24] G. J. O. Vermeer, "3-D symmetric sampling," Geophysics, vol. 63, no. 5, pp. 1629-1647, 1998.

[25] N. Cooper, "A world of reality—designing land 3D programs for signal, noise, and prestack migration," The Leading Edge, vol. 23, no. 10, pp. 1007-1014, 2004.

[26] B. Heath, "Weighing the role of cableless and cable-based systems in the future of land seismic acquisition," First Break, vol. 28 , no. 6,10 pages, 2010 . 

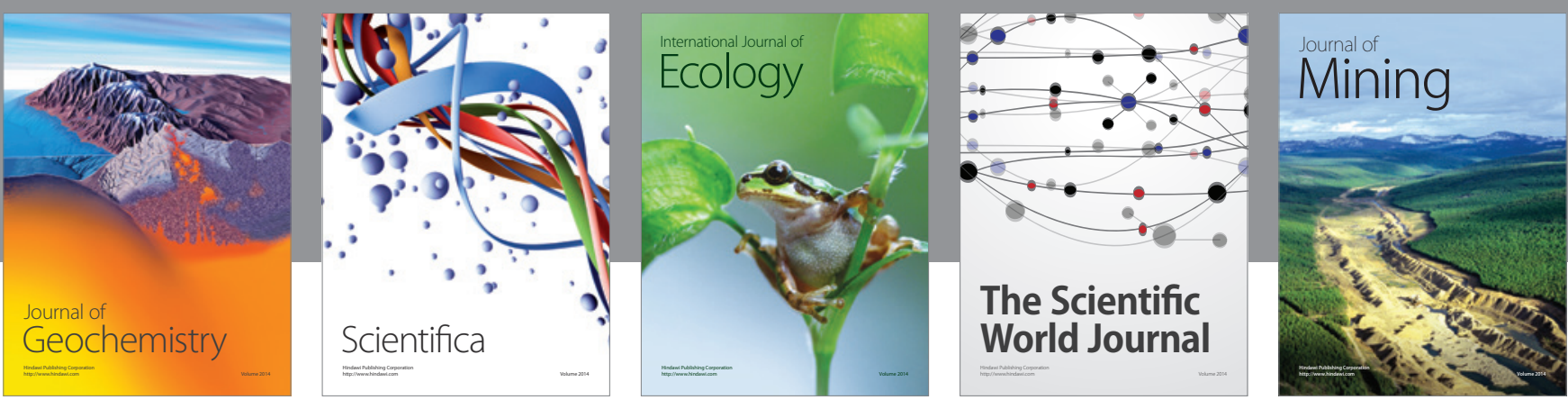

The Scientific World Journal
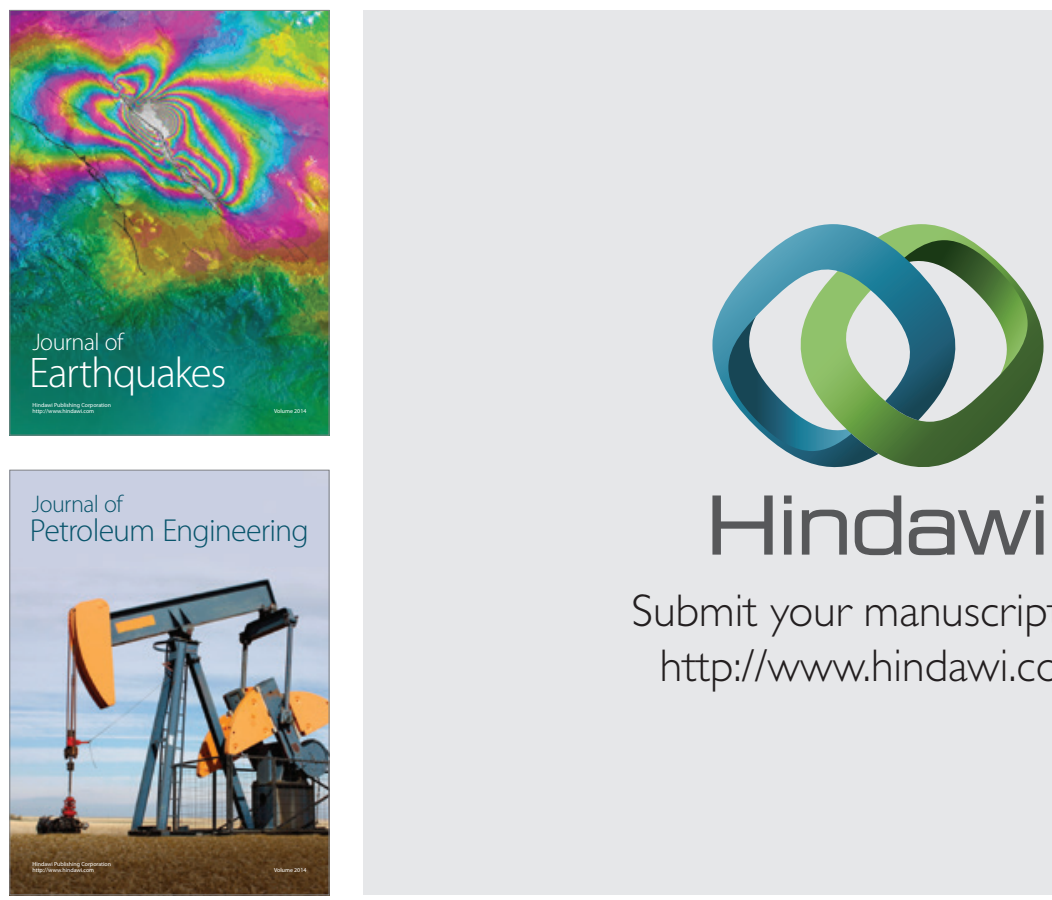

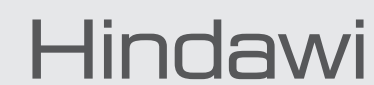

Submit your manuscripts at

http://www.hindawi.com
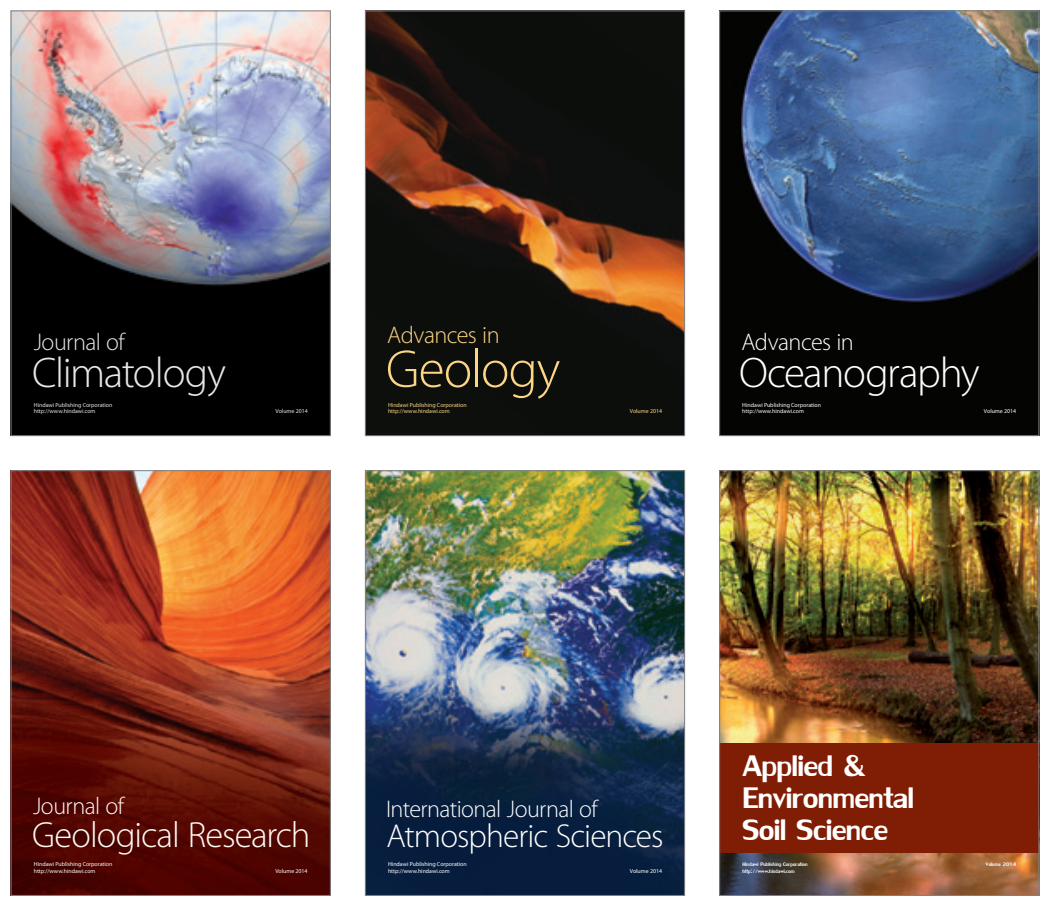
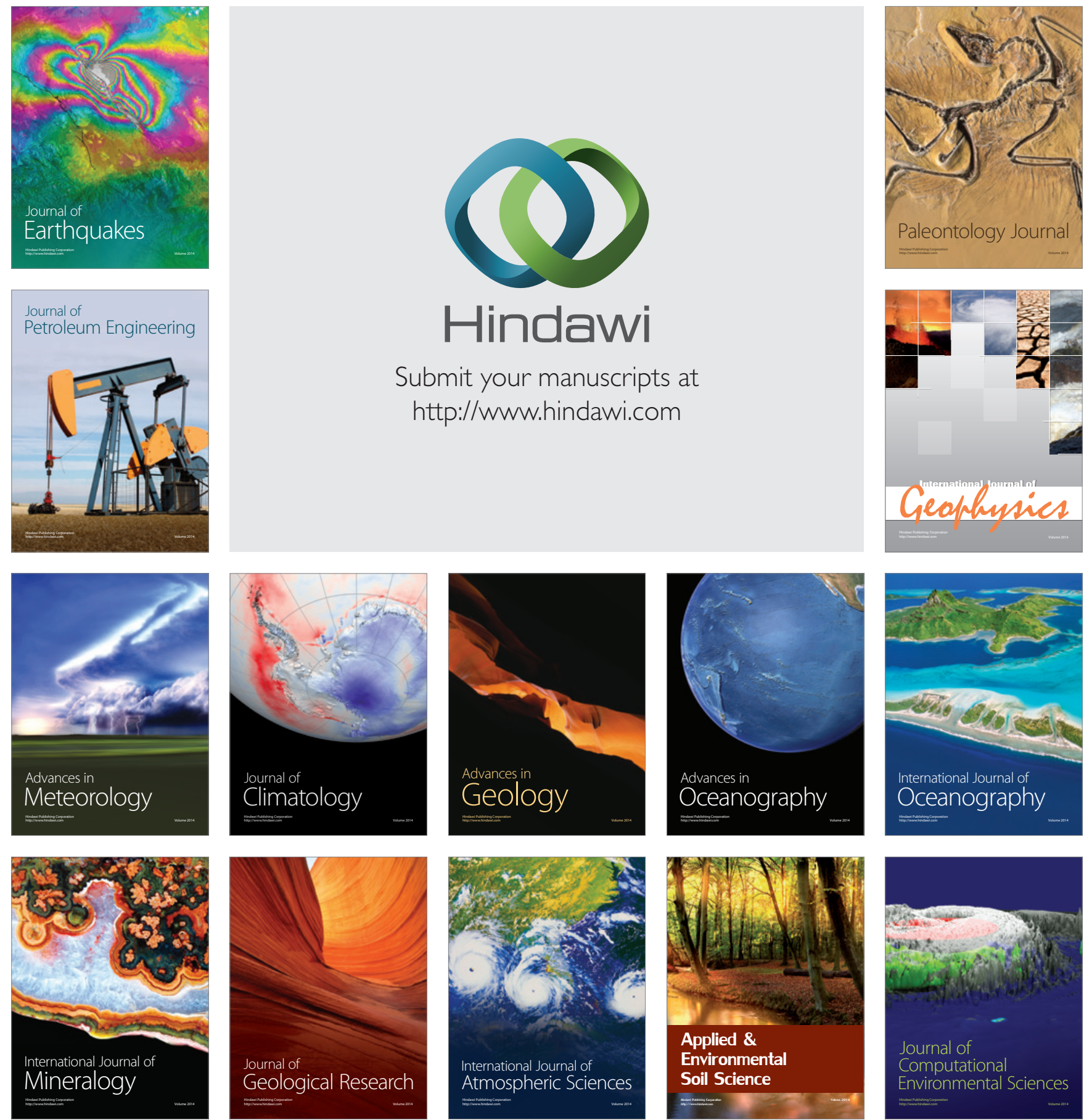